\title{
Best practices for measuring emerging light-emitting diode technologies
}

Miguel Anaya ${ }^{1}$, Barry P. Rand², Russell J. Holmes ${ }^{3}$, Dan Credgington ${ }^{1}$, Henk J. Bolink ${ }^{4}$, Richard H. Friend $^{1}$, Jianpu Wang ${ }^{5}$, Neil C. Greenham ${ }^{1}$, Samuel D. Stranks ${ }^{1 *}$

${ }^{1}$ Cavendish Laboratory, University of Cambridge, UK.

2 Department of Electrical Engineering \& Andlinger Center for Energy and the Environment, Princeton University, USA.

${ }^{3}$ Department of Chemical Engineering and Materials Science, University of Minnesota, USA.

${ }^{4}$ Instituto de Ciencia Molecular, Universidad de Valencia, Spain.

${ }^{5}$ Institute of Advanced Materials, Nanjing Tech University, China.

*sds65@cam.ac.uk

Emerging light emitting devices based on organic semiconductors (OLEDs), inorganic quantum dots (QLEDs) and metal-halide perovskites (PeLEDs) are pushing current solid-state lighting into a new era in which light can be manipulated in a number of ways. The unprecedented control over colour, brightness and directionality of these emerging light sources, in addition to inexpensive fabrication routes, has opened up a world of new applications, with OLEDs already showing a growing presence in display markets. Historically, the adoption of standardised protocols by the LED community to assess the performance of new materials and architectures has provided emerging LED technologies with the necessary self-reliance to mature..$^{1,2,3}$ The recent development of disruptive concepts in OLEDs and QLEDs, ${ }^{4,5}$ and the arrival of very new technologies such as PeLEDs - based on a diverse class of emitters such as 3D, 2D, OD or double perovskites, $6,7,8$ - has aroused great excitement about further potential impact in the lighting and display industries. However, these findings at the laboratory stage are often not described in a consistent and uniform way, owing in large part to a range of peculiar phenomena in these emerging technologies such as transient effects. This leads to challenges in making real comparisons between reported devices, difficulties with reproducibility, and a lack of clarity regarding the realistic technology readiness level of new concepts and technologies. Next-generation solar cells, for example those based on perovskite absorbers, have recently transitioned through a similar "coming of age" as they have become more competitive with incumbent technologies. This has been aided by the clear standards in the photovoltaics field that are broadly followed by both the academic and industrial research communities. In this Comment, we highlight key considerations for reporting the characterisation of new LED technologies, in particular drawing on historical guidelines to discuss how these approaches should be used and interpreted in the context of such new LED technologies. Furthermore, we demonstrate typical calculation procedures in the Supplementary 
Information (SI). By doing so, we propose guidelines for reviewers, experimenters and industry alike with the aim of facilitating rationalised and precise comparison between novel LED technologies utilising different emitter classes and architectures.

\section{$\underline{\text { Quantum efficiencies }}$}

We first define the most important metrics to determine LED performance. The external quantum efficiency (EQE) is defined as the ratio of photons that escape the LED to the number of electrons flowing in the external circuit. For many applications, EQE is defined specifically for photons that escape into the forward viewing hemisphere, as defined by Forrest et al. ${ }^{2}$ To properly quantify the EQE at a given current density $(\mathcal{J})$, there are three approaches, each of which is in principle equivalent provided the measurements are performed carefully with appropriate calibration. A first approach utilises a large photodetector at close proximity to the LED to collect the photons emitted in the forward hemisphere (Figure 1a). Here, the measurement design (distance and geometry) must ensure a good compromise between detection of light, by maximising collection solid angle, and mitigation of detection inaccuracies such as reflection losses at small angle or edge effects in the photodetector. The detector should not be in contact with the device, which would otherwise lead to a modification of light out-coupling and reflectance losses by altering the device/air interface. A second approach to measure EQE is by collecting all photons in the forward-emitting hemisphere through use of an integrating sphere in a $2 \pi$ geometry and a spectrometer (Figure 1b).

A third approach is to place the photodiode at a large distance compared to the dimensions of the emitting area and the thickness of the device (Figure 1c; see SI for example calculations). This approach might be desired for applications in which the forward-emitting direction (perpendicular to the substrate) is most important, such as for displays. Although it must be accurately determined, the exact solid angle subtended by the photodetector is not strictly important provided that both the response of the photodetector to light arriving at different angles of incidence, and the directionality of emitted light from the LED, are known; Lambertian emission profiles are commonly observed for most practical LED devices. Given the potential for significant systematic error in these assumptions, one can perform a measurement to confirm the angular distribution of the emission, particularly if novel architectures and/or emitters are employed, or for devices that have substantial scattering due to layer roughness, among other factors. Since the Lambertian assumption may not hold in these cases, the simple use of a photodetector subtending a small solid angle could conceal any substantial emission away from the 
perpendicular direction for superlambertian emitters or overestimate the EQE value in sublambertian emitters. For this reason, one can employ a photodetector mounted on a goniometer with the aim of determining the full angular distribution of emitted photons (see Figure 1c). To eliminate any influence from the angular dependence of detection, the photodetector should subtend as small a solid angle as practical.

a)

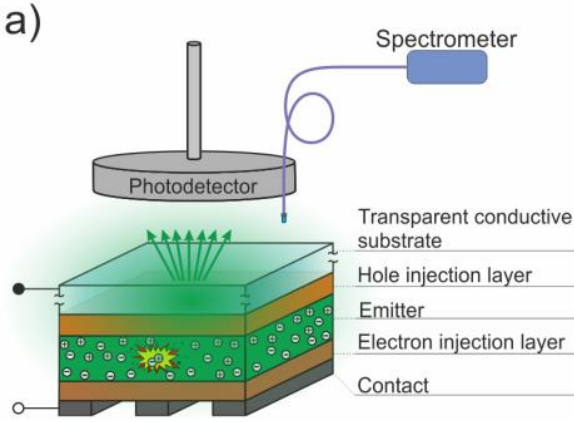

b)

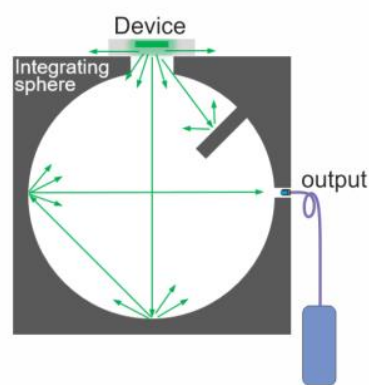

c)

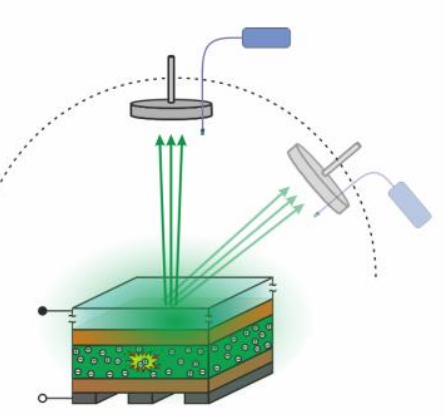

Figure 1. Proposed characterisation setups for emerging LEDs. (a) A typical EQE measurement in which a photodiode placed at an appropriate distance from the device is used to measure emitted photons. Along with charge injection characteristics (i.e. current and voltage), this is used to determine the radiance and luminous efficacy. A spectrometer is used to extract spectral information. (b) A direct EQE measurement by using an integrating sphere and a spectrometer. (c) A photodetector mounted on a goniometer to determine the full angular distribution of emission.

In order to accurately determine the emission quantum efficiency in each case, it is essential to account for the spectral-dependent losses caused by any element of the measurement setup placed between the LED and the photodetector (e.g. lenses, filters, optical fibers, gratings, mirrors, sample holder). Likewise, one must perform a careful spectral response calibration of the photodetector at the device emission wavelengths; this of course first necessitates a measurement of the electroluminescence (EL) spectrum using a spectrometer (cf. Figure 1). In the case of emitters having a broad emission spectral spread, the average responsivity of the photodetector weighted by the emission spectrum should be used, as outlined by Forrest et al (see also SI). ${ }^{2}$ One may also consider the use of a well-calibrated spectroradiometer as an alternative to a calibrated photodetector, and one can then determine EQE through accurate measurement of the device active area; this approach is widely employed in industry. In any case, any measurement setup including detector must be carefully and regularly calibrated. We also advise the use of well-aligned aperture masks to avoid detecting waveguided emission contributions from the substrate edges or light scattered out from beyond the pixel area, though care must be taken to account for the thickness of the glass and to not interfere with the escape cone, which can be a particular issue for measuring the angular dependence. Furthermore, cross-checking EQE 
measurements using two different methods, for example through use of a photodetector via the first method and a spectroradiometer via the third method, would be good practice to validate the measurement and calibration procedures. This approach would also help to mitigate any significant errors arising, for example, through errors in the measurement of the distance between the detector and device active area that may result in large errors in the calculated EQE. In the interests of transparency, research groups should carefully describe their device design, measurement geometry, and calibration approaches; it would be good practice to also make their calibration files available through open repositories or supporting information files in publications. In principle, any of the aforementioned characterisation protocols to report quantum efficiencies are equivalent as long as they are conducted correctly and transparently. Quantum efficiency values must be quoted in context with the carrier density (ascertained through proxies such as luminance or current density) under which they are measured. Ideally, the dependence of the EQE should be provided across several orders of magnitude of luminance or current density to understand the emission characteristics and limitations including current leakage, peak EQE and "roll-off" (aka "droop").

The internal quantum efficiency (IQE) is defined as the ratio of photons generated within the device to the number of charge carriers injected. It is related to the EQE through the extraction efficiency, which is the ratio of photons escaping the device to those generated. There are a plethora of internal optical processes occurring within the device such as waveguiding, reabsorption, surface plasmon losses and multi-layer reflections, among others, which render this ratio difficult to calculate; detailed knowledge of device interfaces and optical properties are therefore essential in order to best estimate the IQE. To this end, it is prudent to use a properly sized integrating sphere in a $4 \pi$ geometry, that is, in which the device is mounted in the centre to collect emitted photons at an output port of the sphere. One must then take care to calibrate the measurement to account for internal losses within the device stack; one way to do so would be to perform an identical measurement but placing a "test" LED in the integrating sphere with exactly the same absorption and reflection spectra as the device under investigation. Accurate calibration and modelling must take into account absorption and reflection losses from parasitic elements in the light path (e.g. device backside, material between pixels) but also model how other internal processes affect light out-coupling. Furthermore, the performance of some novel LED structures are substantially influenced by additional internal processes such as efficient photon recycling, for example in many of those based on crystalline semiconductors, including the halide 
perovskites, which exhibit small Stokes' shifts between absorption and emission energies. These phenomena have an important impact on both the IQE and EQE because the opportunity for forward emission is substantially increased due to the larger number of absorption and re-emission events taking place within the device..$^{8,9}$ Similarly, any potentially anisotropic transition-dipole moment orientation, as is the case in many OLED devices, must also be taken in to account when calculating the extraction efficiency. ${ }^{10}$ Further information about the internal processes of the device can be ascertained by correlating the EL and EQE (IQE when provided) with their photo-induced counterparts - photoluminescence (PL) and photoluminescence quantum efficiency (PLQE). Careful comparisons between these quantities in a full device stack configuration allows identification of injection issues, the specific location (e.g. depth) of recombination within the device, and nonradiative recombination processes taking place in both standalone emissive films and interfaces. ${ }^{9}$ Efforts to quantify the IQE and extraction efficiency precisely are needed to unravel the working principles of the LED and understand how close a particular emitter and device stack combination is to maximum performance.

\section{Device emission colour and intensity}

In order to assess the emissivity of an LED under operating conditions, one should estimate the radiance $\left[\left(\mathrm{W} \cdot \mathrm{sr}^{-1} \cdot \mathrm{m}^{-2}\right]\right.$, which corresponds to the power from photons emitted per unit area per unit solid angle. Nevertheless, most LED applications require one to account for the perceived emission colour of the LED in the visible range of the electromagnetic spectrum. This is typically done by recasting radiance as luminance $\left[\mathrm{cd} / \mathrm{m}^{2}\right]$ and $E Q E$ in terms of current efficacy $[\mathrm{cd} / \mathrm{A}]$, by weighting the emitted photon energies by the photopic response of the human eye (see SI). These metrics as a function of current or voltage are critical for understanding to what extent the device is relevant for applications such as displays or lighting. Indeed, from a technological viewpoint, outstanding EQE is only meaningful if it can be realized at practical luminance values. A typical operating value of luminance for a given colour for display applications is at least $100 \mathrm{~cd} / \mathrm{m}^{2}$, but often above $1000 \mathrm{~cd} / \mathrm{m}^{2}$ for OLED displays and other lighting applications. ${ }^{11,12,13}$ By contrast, when considering LEDs with predominantly UV or NIR electroluminescence, one should refer directly to the measured radiance due to the very low photopic response at these wavelengths; similarly, one should avoid inverting measured photometric units (such as those measured through a spectroradiometer) for spectral regions outside the calibrated range or where the photopic response tends to zero. We also note here that using "low turn-on" voltage in a 
subjective way should be discouraged, and instead suggest reporting the voltage corresponding to a reasonable emission output, for example luminance on the order of 1 or $10 \mathrm{~cd} / \mathrm{m}^{2}$ or the equivalent radiance for non-visible emitters. A useful metric for applications where electrical power consumption is important (e.g. lighting), i.e. accounting for both current and voltage, is the luminous efficacy [Im/electrical W]; this metric also integrates emitted photons across all angles.

The colour of the emission (e.g. peak wavelength) and its quality (e.g. full width at half maximum) is given by the EL spectrum. One can precisely define colour using the chromaticity coordinates $(x, y)$ established by the Commission Internationale de l'Eclairage (CIE).${ }^{14}$ These are obtained through the tristimulus values $X, Y$ and $Z$, which reproduce the behaviour of the three different cone cells present in the human eye, and are widely employed to judge the suitability of a light source for the application pursued. In the case of white lighting, one can use the spectrum emitted by an ideal black body radiator as a reference to assign the correlated colour temperature (CCT) [K]. In addition, for illumination purposes, the colour rendering index (CRI) quantifies how well a given light source reproduces the colour of illuminated objects versus an ideal black body light source..$^{15}$ We note that the multi-layered thin film character of emerging LEDs, and the integration of optically active structures to control light out-coupling, can lead to variations of the spectral output (colour) of the device with the angle of emission.

\section{Stability and device lifetime}

Evaluating the lifetime of emerging LEDs is critical, particularly for technologies that typically suffer from substantial changes in performance over operation time. The following approaches can assess performance in a reasonable manner at the laboratory scale; once emerging LEDs demonstrate sufficient laboratory stability, they would then need to pass more demanding industry-standard stress tests. $^{3}$

Researchers should first ensure that their devices emit light long enough to be measured and that their stability over the measurement duration has minimal impact on the extracted device performance parameters. For this, we propose considering the following simple procedures. Current-voltage scans should be carried out at two different rates; first a rapid scan (e.g. steps of $0.5 \mathrm{~V}$ ), and then a slow scan (e.g. steps of 0.1 or $0.01 \mathrm{~V}$ ) from low to higher voltages and back. Measurements should be checked to ensure that the device current-voltage-luminance characteristics are not evolving during acquisition 
at a given voltage bias. Asynchronous readout of current and radiance can lead to significant errors in calculated EQE. The backwards scans will also allow one to decouple whether the maximum achievable brightness and subsequent roll-off at higher operational conditions occurs because of degradation or changes in recombination regime (e.g. due to non-radiative Auger recombination outcompeting radiative processes at high carrier densities). The EL spectrum should ideally be confirmed at the maximum EQE as well as at the maximum achievable brightness, and consideration given for any resulting change in photodetector response. Use of a spectroradiometer may give a more reliable estimation of the EQE than a photodetector if the EL spectrum is changing over time during characterisation.

If a significant claim of a piece of research is a long-term stability enhancement, such reports must appropriately capture the operational stability of the devices over time. For this, EQE, or a proxy such as luminance, should be presented as a function of time at a constant operational condition relevant to the application, for example constant current density corresponding to a starting luminance of 100 $\mathrm{cd} / \mathrm{m}^{2}$. It would be useful to also present results at both lower (e.g. starting at $10 \mathrm{~cd} / \mathrm{m}^{2}$ ) and higher $\left(1000 \mathrm{~cd} / \mathrm{m}^{2}\right)$ operational conditions to learn more about the limitations of the operating device. Typical metrics for this are the "T50" and "T95" values, or similar, which reflect the time taken by the LED to drop to $50 \%$ and $95 \%$ of their initial output, respectively. We strongly discourage the extrapolation of lifetimes extracted from measurement at one operating condition to a different operating condition; operating lifetime must always be directly measured unless lifetimes are so long that this is impractical. In this latter case, acceleration factors, for example acquired by operating at elevated current density and/or temperature, would be required to assess device stability in a practical period of time; such factors also yield valuable insight into degradation mechanisms. In addition, researchers should confirm the colour stability of the operating devices. Phase segregation in mixed halide perovskites, nanocrystal aggregation in confined systems and chemical degradation of organic species are examples that lead to colour instabilities (see example in Figure 2). One relevant example is in the case of blue LEDs, where one can overestimate the EQE if the emission shifts to the green during operation, a region at which many photodiodes have better sensitivities. We recommend reporting the EL spectrum of the device before and after the operational stability test. Researchers need not shy away from reporting LEDs incorporating emitting materials that have not yet reached sufficient stability levels, but rather 
these stability deficiencies should be transparently and honestly reported to allow further development by the wider community.

We also emphasise the importance of stating all measurement conditions including environment (humidity, oxygen) and, ideally, temperature. For rigorous stability testing, we recommend maintaining device temperatures in the range between $25^{\circ} \mathrm{C}$ and $35^{\circ} \mathrm{C}$ to ensure typical and reproducible laboratory conditions. Minimising exposure of the device to moisture and oxygen from the surroundings is important due to the detrimental (and, often, unpredictable) effect on material performance. Any packaging or encapsulation protocols must be specified in detail to allow them to be reproduced and adequately compared.

\section{Concluding remarks}

This Comment outlines critical performance measurement considerations for emerging LEDs to ensure best practice. We encourage the reporting of device failures in any of the key parameters since this will open up new research paths to address such issues. Furthermore, researchers should appropriately report the statistics of device performance, including not only the champion performances but also the metrics such as the mean and standard deviation; in this context, one should also clearly state whether the presented statistics relate to the evaluation of several pixels from the same device substrate, from different substrates and/or from independent device batches. Indeed, we urge researchers to report all details of materials synthesis, device fabrication and measurement protocols, no matter how seemingly insignificant, to provide better reproducibility between laboratories. Furthermore, we encourage independent measurement of device performance through external laboratories or industry, for example through round-robin studies, to better validate measured performances. Independent certification centres similar to those existing for photovoltaic technologies may be an interesting concept to explore provided there is sufficient demand, though this may only be needed when devices are approaching performance limits and further efficiency increments require exceptional precision and validation. The proper implementation of the protocols discussed in this Comment will allow the unambiguous characterisation of novel LEDs at the laboratory scale, ultimately leading to more rapid progress in performance and stability towards downstream applications. 
a)

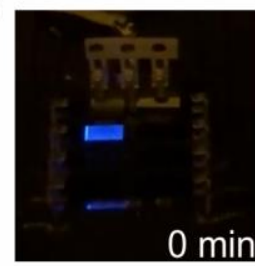

$0 \mathrm{~min}$
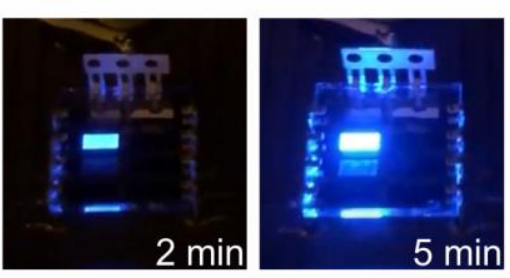

Time

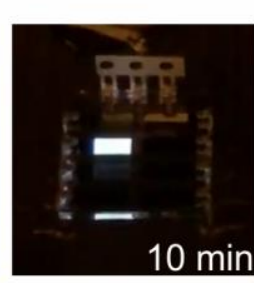

$10 \mathrm{~min}$

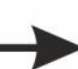

b)

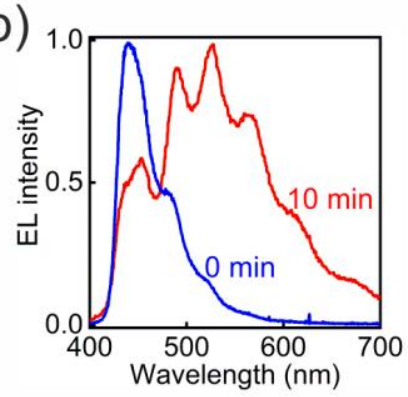

Figure 2. Example of colour instability: from blue to white in a suboptimal TFB-based OLED at a constant bias of $8 \mathrm{~V}$ during 10 minutes exposure in air. (a) Digital camera pictures of the device during degradation. (b) Electroluminescence spectra of the device at the initial and the final stage.

\section{Table. Checklist for papers reporting emerging LEDs.}

i) EQE vs $J$ and, if appropriate, luminous efficacy vs $J$ or luminance.

ii) $J$ and luminance/radiance vs $V$ (carefully monitoring for any transient changes); comment on maximum achievable brightness and roll-off.

iii) EL spectrum, wavelength of the peak maximum and FWHM.

iv) Colour coordinates (CIE). Only applies for visible emission.

v) Correlated colour temperature (CCT) and colour rendering index (CRI). Only applies for white-light LEDs.

vi) Full details on calibration protocols, measurement setup (physical dimensions of the device and the detector, and the distance between them) and assumptions made regarding emission/collection profiles.

For reports focussing on long-term stability:

vii) Operational stability. Emitted photons over time vs constant $J$ (or constant detection).

viii) Colour stability. $E L$ spectrum at $\mathrm{t}=0$ and $\mathrm{t}=$ final for fixed $J$.

1 Greenham, N. C., Friend, R. H. \& Bradley, D. D. C. Adv. Mater. 6, 491-494 (1994).

2 Forrest, S. R., Bradley, D. D. C. \& Thomson M. E. Adv. Mater. 15, 1043-1048 (2003).

3 Methods approved by the Illuminating Engineering Society of North America (IESNA): LM-79-08, LM-

80-08, TM-21-11, and LM-82-12.

$4 \mathrm{Ai}, \mathrm{X}$. et al. Nature 563, 536-540 (2018).

5 Yang, Y. et al. Nature Photonics 9, 259-266 (2015).

6 Cao, Y. et al. Nature 562, 249-253 (2018).

7 Lin, K. et al. Nature 562, 245-248 (2018).

8 Zhao, B. et al. Nature Photonics 12, 783-789 (2018).

9 Stranks, S. D., Hoye, R. L. Z., Di, D., Friend, R. H. \& Deschler, F. Adv. Mater. 1803336 (2018). 
10 Kim, K.-H. et al. Nat. Commun. 5:4769 (2014).

11 Lee, E. et al. J. Inf. Disp. 17, 151-158 (2016).

12 Light and lighting. Lighting at work places. European Standard EN 12464-1:2011 and EN 124642:2014.

13 US General Services Administration, GSA, 6.15 Lighting, 2014.

14 Commission Internationale de l'Eclairage, Colorimetry, 3rd edition, CIE 15:2004.

15 Commission Internationale de l'Eclairage, Method of Measuring and Specifying Colour Rendering of Light Sources, CIE 13.3-1995. 


\section{$\underline{\text { Acknowledgements }}$}

S.D.S. and M.A. acknowledge funding from the European Research Council (ERC) (grant agreement No. 756962 [HYPERION]) and the Marie Skłodowska-Curie actions (grant agreement No. 841386) under the European Union's Horizon 2020 research and innovation programme. S.D.S acknowledges support from the Royal Society and Tata Group (UF150033). The authors acknowledge the EPSRC (EP/M005143/1). B.P.R. acknowledges support from the Air Force Office of Scientific Research under Award no. FA9550-18-1-0037. R.J.H. acknowledges support from the a Visiting Fellowship at Clare Hall and a Leverhulme Trust Visiting Professorship, both at the University of Cambridge, the National Science Foundation (ECCS-1509121 and DMR-1708177), and the University of Minnesota Institute on the Environment. D.C. acknowledges funding from the Royal Society. H.J.B acknowledges financial support from the Spanish Ministry of Economy and Competitiveness (MINECO) via the Unidad de Excelencia María de Maeztu MDM-2015-0538 and MAT2017-88821-R. The authors thank Patrick Conaghan for providing data for the example LED calculations. 